\title{
Transport in porous media
}

\author{
Omid Dashtpour ${ }^{1}$, Hooman Fallah ${ }^{2 *}$ \\ ${ }^{1}$ National Iranian oil company (NIOC), National Iranian Drilling Company (NIDC), Iran \\ ${ }^{2}$ Islamic Azad University, Firoozabad Branch, Firoozabad, Iran
}

\section{Email address:}

omidnafti@yahoo.com(O. Dashtpour), hooman.fallah@gmail.com(H. Fallah)

\section{To cite this article:}

Omid Dashtpour, Hooman Fallah. Transport in Porous Media. International Journal of Oil, Gas and Coal Engineering. Vol. 1, No. 1, 2013, pp. 1-6. doi: 10.11648/j.ogce.20130101.11

\begin{abstract}
Transport through porous medium occurs in numerous processes of environmental, chemical, petroleum and civil engineering. A lot of investigations have been done in order to understand the mechanisms of the transport of particulate suspension flow through porous medium. Transport of particulate suspensions and colloids in porous media is accompanied by particle capture and consequent permeability decline. In general, deep bed filtration studies have been conducted to analyse the mechanism involved in the processes of capturing and retaining particles occurs throughout the entire depth of the filter and not just on the filter surface. In this work, the steady-state transport equation is presented and the solution to the complete advective-dispersion equation for particulate suspension flow has been derived for the case of a constant filter coefficient. This model includes transport parameters which are particle advective velocity and particle longitudinal dispersion coefficient. This theoretical investigation of the transport of particles flowing in porous media is limited to flows with low Reynolds number (linear and laminar flow) and high Peclet number.
\end{abstract}

Keywords: Porous Media, Particle Advective Velocity, Longitudinal Dispersion Coefficient, Filter Coefficient

\section{Introduction}

Transport through porous medium occurs in numerous processes of environmental, chemical, petroleum and civil engineering. Transport of particulate suspensions and colloids in porous media is accompanied by particle capture and consequent permeability decline.

It occurs in oil reservoirs during sea or produced water injection, drilling fluid invasion causing formation damage, filtration of completion fluid, fines migration during production of heavy oils in low consolidated reservoirs resulting in productivity.

Prediction of particle propagation and retention by mathematical modeling is an essential stage during planning and design of above-mentioned industrial processes. It helps in design of injected water treatment, in particle sizing of drilling and completion fluids, in sizing the gravel pack and designing the sand screens.

In general, deep bed filtration studies have been conducted to analyse the mechanism involved in the processes of capturing and retaining particles occurs throughout the entire depth of the filter and not just on the filter surface [1].

In this work, the steady-state transport equation is presented and the solution to the complete advective-dispersion equation for particulate suspension flow has been derived for the case of a constant filter coefficient. This model includes transport parameters which are particle advective velocity, particle longitudinal dispersion coefficient and filter coefficient. The numerical model needs to be developed for general case of a transition filter coefficient. The individual parameters are analysed according to dimensional analysis argument.

Here, a particle mass transport equation is developed which includes the transport parameters, advective velocity and longitudinal dispersion coefficient. A solution to the complete advective-dispersion equation for particle transport was derived for the case of constant filter coefficient. The individual parameters are analysed according to dimensional analysis argument.

In order to develop the theories understanding of the basics of flow in porous media is needed advective fluid velocity and longitudinal dispersion coefficient.

This theoretical investigation of the transport parameters of particles flowing in porous media is limited to flows with low Reynolds number (linear and laminar flow) and high Peclet number (advection dominates diffusion).

The theoretical development used dimensionless numbers to define the transport parameters and incorporated them into an advective-dispersion equation describing particle 
transport.

\section{Advective Velocity}

The averaged particle velocity in the porous media, has been found to be either the same or slightly higher than that of the carrier fluid [2]. This deviation is caused by the particle's size. The expected difference can be determined by analyzing the velocity profiles of both the fluid and the particles in a pore [3]. The model has been formulated for a capillary tube which has a constant rate with the following assumption: No interactions between the particles and the wall, suspension is well-mixed with a constant concentration across the cross section. There is no transverse flow.

$$
\begin{gathered}
U_{S}=U_{O}\left\{1-\left(\frac{r}{r_{o}}\right)^{2}\right\} \\
\left(0<r<r_{o}\right) \\
U_{P}=U_{O}\left\{1-\left\{\frac{r}{r_{o}}\right\}^{2}-\gamma\left\{\frac{a_{p}}{r_{o}}\right\}^{2}\right\}\left(0<r<r_{o}-a_{p}\right)
\end{gathered}
$$

As the particle travels through a tube, Brownian motion and shear action will cause the particle to travel across the entire cross-section of the tube except that the center-line of the particle will be excluded from the immediate region of the wall due to its radial dimension.

After the particle has traveled far enough longitudinally through the tube, the particle will have spent equal amounts of time in all radial position across the capillary tube [4].

Integration of the velocity profile over the range of possible radii shown in Eq. (1) for both the particle and fluid yields the higher average velocity for the particle than that for the carrier fluid.

The average fluid velocity, $V_{S}$, in a capillary tube is:

$$
V_{S}=\frac{U_{O}}{2}
$$

The average velocity of a particle, $V_{P}$, in a capillary tube is:

$$
V_{P}=U_{O}\left(1-\frac{1}{2}\left\{1-\frac{a_{p}}{r_{o}}\right\}^{2}-\gamma\left\{\frac{a_{p}}{r_{o}}\right\}^{2}\right)
$$

By inspecting Eq. (2) and (3), the particles are expected to have a larger average velocity than the carrier fluid velocity. This enhanced velocity of the particle can be expressed as a fractional difference between the two average velocities:

$$
\Delta V=\frac{V_{P}-V_{S}}{V_{S}}=2 \frac{a_{p}}{r_{o}}-\frac{7}{3}\left(\frac{a_{p}}{r_{o}}\right)^{2}
$$

This equation shows that as the radius of the particle increase, the difference between the average particle velocity and the average fluid interstitial velocity also increase. This increase is not unbounded but reaches a maximum $\Delta V$ value for $\frac{a_{p}}{r_{o}}=\frac{3}{7}$; as $\frac{a_{p}}{r_{o}}>\frac{3}{7}$ the velocity difference decrease.

In physical sense, the pore radius can be estimated to approximately equal to one-fifth of media grain diameter $\left(r_{o}=\frac{1}{5} d_{g}\right)$; therefore the largest possible particle to be able to fit through the porous bed has a radius to this pore radius $\left(a_{p}=r_{o}\right)$.

For a particle with $a_{p}=r_{o}$, the particles have been shown to collect on the bed surface in a cake [4].

These references show that the onset of deep bed filtration occurs for a particle radius $a_{p}$ less than one-twentieth of the media grain diameter $\left(a_{p}<\frac{1}{20} d_{g}\right)$. Particles with the radii larger than this will not transport into the bed, but will collect on the surface. By letting $d_{g}=5 r_{o}$, the largest particle which will transport has a radius equal to one-fourth of the pore radius $\left(a_{p} \leq \frac{1}{4} r_{o}\right)$.

\section{Longitudinal Dispersion Coefficient}

An important element of any dispersion model is the representation of the geometry of the porous medium. Houseworth [5] has thoroughly reviewed such longitudinal dispersion model for solute tracers.

Instead of modeling the internal structure of a porous medium, dimensional analysis is used to analyze the problem. In this study, the effect of mechanisms is expected to scale with the pertinent transport variables.

The pertinent variables for solute dispersion are:

$$
D_{L}=\text { longitudinal dispersion coefficient ; }
$$

$D$

= free fluid molecular diffusion coefficient of solute $V_{S}=$ fluid interstitial velocity $;$

and $d_{g}=$ media grain diameter $(L)$.

From the Buckingham pi theorem, the following pairs of groups are formed:

$$
\begin{gathered}
\frac{D_{L}}{V_{S} \cdot d_{g}}=F\left[\frac{V_{S} \cdot d_{g}}{D}\right], \text { or alternatively, } \\
2 \cdot \frac{D_{L}}{D}=F\left[\frac{V_{S} \cdot d_{g}}{D}\right]
\end{gathered}
$$

where: Pe $=$ Peclet number $=\frac{V_{S} \cdot d_{g}}{D}$

Experimental data for solute longitudinal dispersion in uniform media show good correlation with these dimensionless groups [5], [6].

When the Peclet number is grater than 1, the two groups can be reduced to one:

$$
\frac{D_{L}}{V_{S} \cdot d_{g}} \approx \text { Constant }
$$

Where: $\mathrm{P} e_{D}=$ dynamic Peclet number $=\frac{V_{S} \cdot d_{g}}{D_{L}}$.

An order to magnitude approximation for the longitudinal dispersion coefficient for solutes can be made with: 


$$
D_{L}=V_{S} \cdot d_{g}
$$

Particle longitudinal dispersion is expected to be similar to that of solutes.

Currently, no particle breakthroughs have been performed by others form which particle longitudinal dispersion coefficient be determined.

\section{Transport of Fluid through Porous Medium}

In this part, the steady-state transport equation is presented and the solution to the complete advective-dispersion equation for particulate suspension flow has been derived for the case of a constant filter coefficient. This model includes transport parameters which are particle advestive velocity and particle longitudinal dispersion coefficient [7].

These parameters have been defined by dimensional analysis using the pertinent variables of the porous media system.

\subsection{Particle Advective Velocity}

The result of the size exclusion for particles flowing in capillary tube can be written as equation (3) where $\gamma=\frac{2}{3}$. By using the equations (2) and (4) we will have:

$$
\begin{gathered}
V^{*}=1+\Delta V \\
\text { where: } V^{*}=\frac{V_{P}}{V_{S}}
\end{gathered}
$$

As the particle size increase, the difference between particle velocity and fluid velocity increase.

\subsection{Particle Longitudinal Dispersion Coefficient}

The previous stated method uses a single velocity and length scale and does not model the internal structure of porous medium. In modeling particle dispersion, the following variable substitutions are used:

$$
\begin{gathered}
D \rightarrow D_{P} \\
V \rightarrow V_{P} \\
D_{L} \rightarrow D_{L P}
\end{gathered}
$$

where: $D_{L P}=$ particle dispersion coefficient ; $D_{P}=$ particle molecular diffusion coefficient; $V_{P}=$ particle velocity; $d_{g}=$ media grain diameter $(L) ;$ and $d_{p}=$ particle diameter $(L)$.
Particle size variable can be removed by using the particle properties as shown, provided $\frac{d_{p}}{d_{g}}<<1$. also the effect of particle size is included in the enhanced advective velocity for the particles.

This analysis shows that particle and solute longitudinal dispersion are similar. When the particle Peclet number $\left(P e_{p}=\frac{V_{P} d_{g}}{D_{P}}\right)$ is grater than 10 , the two groups can be reduced to one :

$$
\begin{gathered}
\qquad \frac{D_{L P}}{V_{P} d_{g}}=\text { constant } \\
\text { where: } P e_{p}=\frac{V_{P} d_{g}}{D_{P}} \\
=\text { particle dynamic Peclet number. }
\end{gathered}
$$

An order of magnitude approximation for the longitudinal dispersion coefficient for particles can be made with:

$$
D_{L P}=V_{P} d_{g}
$$

As mentioned before, the dimensional argument for defining the longitudinal dispersion coefficient is only valid when $\mathrm{Pe}_{\mathrm{p}} \gg 1$. For uniform media, this restriction is seen to be $\mathrm{Pe}_{\mathrm{p}}>10$ [5].

Flow conditions are simultaneously limited to the linear, laminar regime for which the Reynolds number must be less than 10 .

\subsection{Steady-State Transport in Porous Medium Equation and Solution}

Particle removal or filtration occurs as a particle suspension flows through a porous medium due to the interaction of the advecting particles and grains of the medium. Iwasaki [8] is credited with being the first to express filtration a first-order decay of particle concentration with distance:

$$
\begin{gathered}
\frac{\partial C}{\partial x}=-\lambda C \\
\text { 1. } C(x=0)=C_{0}
\end{gathered}
$$

And a solution in dimensional terms:

$$
C=C_{0} \exp [-\lambda x]
$$

Or, in dimensionless terms:

$$
\begin{aligned}
& C^{*}=\exp \left[-\lambda^{*} x^{*}\right] \\
& \text { where: } C^{*}=\frac{C}{C_{0}} \\
& C_{0}=C(x=0)
\end{aligned}
$$




$$
\begin{aligned}
& \lambda^{*}=\lambda d_{g} ; \\
& x^{*}=\frac{x}{d_{g}} ;
\end{aligned}
$$

A complete equation of steady-state filtration can be formulated by using the general steady-state advection-dispersion equation of transport for particle concentration with a sink term to describe particle removal due to filtration:

$$
0=D_{L P} \frac{\partial^{2} C}{\partial x^{2}}-V_{p} \frac{\partial C}{\partial x}-V_{p} \lambda C
$$

The following semi-infinite medium boundary conditions are:

$$
\begin{aligned}
& C(x=0)=C_{0} \\
& \lim _{x \rightarrow \infty} C(x)=0 .
\end{aligned}
$$

The solution which is shown in dimensional terms is derived in appendix:

$$
\begin{gathered}
C(x)=C_{0} \exp [\alpha x] \\
\text { where: } \alpha=\frac{1}{2} \frac{V_{P}}{D_{L P}}\left(1-\sqrt{1+4 \lambda \frac{D_{L P}}{V_{P}}}\right)
\end{gathered}
$$

In dimensionless terms the transport equation becomes:

$$
0=\frac{\partial^{2} C^{*}}{\partial x^{* 2}}-P e_{D p} \frac{\partial C^{*}}{\partial x^{*}}-P e_{D p} \lambda^{*} C^{*}
$$

With the same boundary conditions:

$$
C^{*}\left(x^{*}=0\right)=1(30) 2 \cdot \lim _{x^{*} \rightarrow \infty} C^{*}\left(x^{*}\right)=0
$$

And a solution in dimensionless terms:

$$
\begin{gathered}
C^{*}=\exp \left[\alpha^{*} x^{*}\right] \\
\alpha^{*}=\alpha d_{g}=\frac{1}{2} P e_{D P}\left(1-\sqrt{1+\frac{4 \lambda^{*}}{P e_{D P}}}\right)
\end{gathered}
$$

\section{Conclusion}

In this work, a mass balance particle transport equation which includes filtration has been developed. This model includes transport parameters which are particle advective velocity, particle longitudinal dispersion coefficient and filter coefficient. The steady-state transport equation is presented and the solution to the complete advective-dispersion equation for particulate suspension flow has been derived for the case of a constant filter coefficient.
This work recommends to be investigated by particle longitudinal dispersion calculation from experimental data. Besides, the numerical model needs to be developed for general case of a transition filter coefficient.

\section{Appendix}

\section{A.1 Solution Derivation}

Consider the one-dimensional steady-state particle advective-dispersion equation which includes the removal term to account for filtration effects:

$$
0=D_{L P} \frac{\partial^{2} C}{\partial x^{2}}-V_{P} \frac{\partial C}{\partial x}-\lambda V_{P} C
$$

$$
\text { where: } C=\text { concentration; }
$$

$$
x=\text { longitudinal distance }(L) ;
$$

$D_{L P}=$ longitudinal dispersion coefficient for particles;

$V_{P}=$ average particle interstitial velocity ; and

$$
\lambda=\text { filter coefficient }\left(L^{-1}\right) \text {. }
$$

With the following boundary conditions:

$$
\begin{aligned}
& \text { 1. } C(x=0)=C_{0} \text {; and } \\
& \text { 2. } \lim _{x \rightarrow \infty} C(x)=0 .
\end{aligned}
$$

For convenience, the $\mathrm{x}$-variable is allowed to range from negative to positive infinity $(-\infty<x<\infty)$, although the equations are only applied for $\mathrm{x}>0$. this avoids difficulty at $\mathrm{x}=0$, because small dispersion is allowed. In dimensionless form, the transport equation becomes:

$$
0=D_{L P} \frac{\partial^{2} C^{*}}{\partial x^{* 2}}-P e_{D p} \frac{\partial C^{*}}{\partial x^{*}}-\lambda^{*} P e_{D p} C^{*}
$$

$$
\begin{gathered}
\text { where: } C^{*}=\frac{C}{C_{0}} ; \\
C_{0}=C(X=0) ;
\end{gathered}
$$

$$
d_{g}=\text { media grain diameter }
$$

$$
\begin{gathered}
x^{*}=\frac{x}{d_{g}} ; \\
P e_{D p}=\frac{V_{p} d_{g}}{D_{L P}} ; \text { and } \lambda^{*}=\lambda d_{g} .
\end{gathered}
$$

With the same boundary conditions:

$$
\begin{aligned}
& \text { 1. } C^{*}\left(x^{*}=0\right)=1 ; \text { and } \\
& \text { 2. } \lim _{x^{*} \rightarrow \infty} C^{*}\left(x^{*}\right)=0 .
\end{aligned}
$$

In order to derive a solution, try the following as a solution:

$$
C^{*}\left(x^{*}\right)=\exp \left[\alpha^{*} x^{*}\right]
$$


Check the equation (A.3) by substituting into Eq. (A.2), this results in second-degree polynomial in term of $\alpha^{*}$, and two roots of this polynomial are:

$$
\begin{array}{r}
\alpha_{1}^{*}=\frac{1}{2} P e_{D P}\left(1-\sqrt{1+\frac{4 \lambda^{*}}{P e_{D P}}}\right) ; \alpha_{1}^{*} \\
=\frac{1}{2} P e_{D P}\left(1+\sqrt{1+\frac{4 \lambda^{*}}{P e_{D P}}}\right)
\end{array}
$$

Using these two roots, Eq. (A.3) becomes:

$$
C^{*}\left(x^{*}\right)=A \exp \left[\alpha_{1}^{*} x^{*}\right]+B \exp \left[\alpha_{2}^{*} x^{*}\right]
$$

The constants of this equation can be determined by applying the boundary conditions:

$$
\begin{gathered}
\text { 1. } C^{*}\left(x^{*}=0\right)=1=A=B ; \text { and } \\
\text { 2. } C^{*}\left(x^{*} \rightarrow \infty\right)=0=(A)(0)+(B)(+\infty) \\
\therefore A=1 \text { and } B=0 .
\end{gathered}
$$

By substituting these constants into equation (A.4), the solution to equation (A.2) becomes:

$$
C^{*}\left(x^{*}\right)=\exp \left[\frac{1}{2} P e_{D P}\left(1-\sqrt{1+\frac{4 \lambda^{*}}{P e_{D P}}}\right) x^{*}\right]
$$

\section{A.2. Solution Approximation}

Consider an approximation for Eq. (A.5) by simplifying $\alpha^{*}$ :

$$
\alpha_{1}^{*}=\frac{1}{2} P e_{D P}\left(1-\sqrt{1+\frac{4 \lambda^{*}}{P e_{D P}}}\right)
$$

Perform a Taylor series expansion of the radical portion of $\alpha^{*}$ of Eq. (A.6) by considering a function $\mathrm{f}(\mathrm{x})$ :

$$
\begin{gathered}
f(x)=\sqrt{1+x} \\
\text { where: } x=\frac{4 \lambda^{*}}{P e_{D P}} .
\end{gathered}
$$

The Taylor series approximation is:

$$
\sqrt{1+x}=1+\frac{1}{2} x-\frac{1}{8} x^{2}+\frac{1}{16} x^{3}-\frac{5}{128} x^{4} \ldots
$$

Since this series is an alternating series, the truncation error must be less than the first truncated term (absolute values). Substituting the first two terms of the series into Eq. (A.6) for the radical portion of $x^{*}$ yields:

$$
x^{*} \cong \frac{1}{2} P e_{D P}\left(1-\left\{1+\frac{2 \lambda^{*}}{P e_{D P}}\right\}\right)=-\lambda^{*}
$$

Substituting the approximation for $\alpha^{*}$, Eq. (A.7), into the exact steady-state equation:

$$
C^{*}=\exp \left[-\lambda^{*} x^{*}\right]
$$

Using Eq. (A.8), $\lambda^{*}$ can be directly calculated from concentration and position measurements. Also, Eq. (A.8) is the same result that would have been derived by ignoring the dispersion term originally.

\section{A.3 Approximate Versus Exact Solution}

The error using the approximate solution can be determined from the relative error of the two solutions, Eq. (A.3) and (A.8):

$\Delta C^{*}=\frac{C_{a}^{*}-C_{e}^{*}}{C_{e}^{*}}=\frac{\exp \left[-\lambda^{*} x^{*}\right]-\exp \left[\alpha^{*} x^{*}\right]}{\exp \left[\alpha^{*} x^{*}\right]}$

where: $C_{a}^{*}=$ approximate solution; and $C_{e}^{*}=$ exact solution.

Eq. (A.9) can be simplified as follows:

$$
\Delta C^{*}=\exp \left[-x^{*}\left(\lambda^{*}+\alpha^{*}\right)\right]-1
$$

The Taylor series expansion for Eq. (A.10) is:

$$
\exp \left[-x^{*}\left(\lambda^{*}+\alpha^{*}\right)\right]=1-x^{*}\left(\lambda^{*}+\alpha^{*}\right)+\cdots
$$

So, the approximate value for $\alpha^{*}$ can be determined using the Taylor series for $\alpha^{*}$ found in section A.2:

$$
\begin{gathered}
\alpha_{1}^{*}=\frac{1}{2} P e_{D P}(1-\sqrt{1+x})=-\frac{1}{2} P e_{D P}\left(\frac{1}{2} x-\frac{1}{8} x^{2} \ldots\right) \\
\leq-\frac{1}{4} P e_{D P} x+\frac{1}{16} P e_{D P} x^{2}
\end{gathered}
$$

Substitute for $\mathrm{x}$ :

$$
\alpha^{*} \leq-\lambda^{*}+\frac{\lambda^{* 2}}{P e_{D P}}
$$

Add $\lambda^{*}$ to Eq. (A.12) in order to determine the argument of the exponential term of Eq. (A.10) so that Eq. (A.11) can be evaluated:

$$
\alpha^{*}+\lambda^{*} \leq \frac{\lambda^{* 2}}{P e_{D P}}
$$

Substitute this approximation into the series expansion expression, Eq. (A.11), of the exponential term in Eq. (A.10):

$$
\left|\Delta C^{*}\right| \leq\left|1-x^{*}\left(\lambda^{*}+\alpha^{*}\right)-1\right| \leq \frac{\lambda^{* 2} x^{*}}{P e_{D P}}
$$

The absolute value of the error is the absolute value of $\Delta C^{*}$ of Eq. (A.14):

$$
\therefore \mid \text { Error }|=| \Delta C^{*} \mid \leq \frac{\lambda^{* 2} x^{*}}{P e_{D P}}
$$




\section{Acknowledgement}

Authors thank National Iranian oil company (NIOC) and National Iranian Drilling Company (NIDC) for their help and financial support.

\section{Nomenclature}

$d g=$ media grain diameter $(\mathrm{L})$

$d p=$ particle diameter $(\mathrm{L})$

$c=$ suspended particle concentration in carrier fluid

$\sigma=$ particle retained concentration

$k$ det $=$ detachment rate coefficient

$U=$ flow velocity

$U S=$ fluid velocity

$U P=$ particle velocity

$U O=$ fluid centerline velocity

$r=$ radial distance

$r o=$ capillary radius

$a p=$ particle radius

$p=$ dynamic pressure

$x=$ longitudinal distance

$D L=$ longitudinal dispersion coefficient $(\mathrm{L} \cdot \mathrm{T}-1)$

$D=$ free fluid molecular dispersion coefficient of solute

$(\mathrm{L} 2 \cdot \mathrm{T}-1)$

$V S=$ fluid interstitial velocity $(\mathrm{L} \cdot \mathrm{T}-1)$

$P e=$ Peclet number $=S g V . d D L$

$P e D=$ dynamic Pec let number $=S g V \cdot d D L$

$C=$ particle concentration $(\mathrm{M} \cdot \mathrm{L}-3)$

$X=$ longitudinal position (L)

$\lambda=$ filter coefficient $(\mathrm{L}-1)$

$W S=$ particle settling velocity

$V S=$ fluid interstitial velocity

$P p f=$ densities of particle and fluid, respectively

$g=$ gravitational acceleration

$H=$ Hamakar constant (ergs)

$N G=$ gravitional group $=\eta G$
$D L P=$ particle longitudinal dispersion coefficient

$(\mathrm{L} 2 \cdot \mathrm{T}-1)$

$D P=$ particle molecular diffusion coefficient in a free fluid (L2·T-1)

$V P=$ particle velocity $(\mathrm{L} 2 \cdot \mathrm{T}-1)$

\section{References}

[1] R. Farajzadeh, "An Experimental Investigation into Internal Filtration and External Cake Build Up," MSc. thesis, Delft University of Technology (2004).

[2] C.G. Enfield., G. Bengtsson., "Macromolecular Transport of Hydrophobic Contaminants in Aqueous Environments", Ground Water, Vol.26, No.1, 1988, pp. 64-70.

[3] E. A. DiMarzio., C. M. Guttman, "Separation By Flow", Macromolecules, Vol. 3, No. 2, 1970, pp. 131-146.

[4] J.P. Herzig, , D.M. Leclerc, P. Le Goff, "Flow of suspension through porous media -application to deep filtration" J. Ind. Eng. Chem. 65(5), 8-35 (1970).

[5] J.E. Houseworth, "Longitudinal Dispersion in Nonuniform, Isotropic Porous Media," Ph.D. Thesis, W.M. Keck Laboratory of Hydraulics and Water Resources, California Institute of Technology, Report No. KH-R-45, (1984).

[6] Foppen, J.W.A., Schijven, J.F "Evaluation of data from the literature on the transport and survival of Escherichia coil in aquifers under saturated conditions," J.Water Res. 40,401-426 (2006).

[7] J.F. Schijven, S.M. Hassanizadeh, "Removal of viruses by soil passage: overview of modeling processes, and parameters," Crit. Rev. Environ. Sci. Technol. 30(1), 49-127 (2000).

[8] T. Iwasaki, "Some notes on sand filtration," Water Works Ass. 1591-1602, 1937. 